IP Periodica Polytechnica

Mechanical Engineering

\author{
57(1), pp. 3]12 2013 \\ DOI: 10.3311/PPme.7011 \\ Creative Commons Attribution (1) \\ RESEARCH ARTICLE
}

\section{Fretting behavior in three different model support configurations}

Szilárd Kovács

Received 2013-04-30

\begin{abstract}
Fretting damage to fuel rods reduces the service lives of the fuel assemblies and therefore increases reactor operating costs. The future development of fretting-resistant spacer grids necessitates the investigation of fretting behavior by means of model tests. This paper presents the results of such tests carried out to investigate fretting in three different fuel rod support configurations. The tests were performed in water at room temperature in a fretting test stand (referred to below simply as "autoclave”) using test rods with zirconium-alloy cladding tubes. The test rod was excited by means of electromagnet to induce vibration. The depth of the fretting marks and their volume increased as testing progressed. The resulting increase in the grid-to-rod gap caused changes in rod dynamic behavior and in the intensity of rod motion. Fretting rate is affected by rod motion, and the presence of edges at the point of contact between rod and support accelerates fretting wear. Spring design affects not only the degree of fretting but also the time history of the fretting process. Steady-state fretting was identified in the case of rods supported by convex springs without edges at the point of rod-to-support contact. The results of the model tests should provide a better understanding of fretting processes inside the reactor. They should not, however, be used to describe real conditions inside a reactor.
\end{abstract}

\section{Keywords}

fretting wear $\cdot$ fretting depth $\cdot$ fretting volume $\cdot$ fretting time history

Szilárd Kovács

AREVA, AREVA NP, Paul-Gossen-Strasse 100, 91052 Erlangen,, Germany

\section{Introduction}

The fuel deployed in the reactor of a nuclear power plant takes the form of uranium pellets inserted inside fuel rod cladding tubes [1]. The fuel rods are combined into a fuel assembly bundle by means of several spacer grids located at different elevations along the fuel assembly's longitudinal axis. Each spacer grid not only positions the fuel rods laterally, but also maintains a certain spacing between the individual rods (rod pitch). Coolant flow through the fuel assembly induces low-amplitude vibration of the fuel rods [2]. The vibration of interest here is that occurring at the locations where the fuel rods are supported, especially the relative vibration of the two surfaces in contact. The wear on the fuel rod cladding tube and the spacer grid caused by this low-amplitude vibration is known as fretting [3]. Fretting in its most extreme manifestation - namely, as cladding tube perforations [4] - reduces the service lives of the fuel assemblies and increases operating costs. Thus, it can be concluded from this that a reduction in fretting susceptibility can serve to increase fuel assembly service life and reduce operating costs.

One of the most important factors governing fretting development is the condition of support, especially the fit between spacer grid and fuel rod [5]. Under beginning-of-life (BOL) conditions, the spacer springs supporting each fuel rod exhibit a large preload. This initial spring preload decreases with increasing core residence time, primarily due to irradiation. Towards end of life (EOL), or at some point in time between BOL and EOL, a gap may arise between the fuel rod and the spacer spring [6]. The condition of this fit has a large effect on possible movements by the fuel rod. At BOL, the fuel rods can only rotate about the contact surface at the point of support (Fig. 1). A large reduction in this preload force can result in the fuel rod (which is surrounded by several springs and dimples) compressing one of the springs in the spacer cell while simultaneously losing contact with another.

The fuel rod experiences its greatest freedom of movement at EOL or whenever a rod-to-grid gap forms [7]. At a spacer spring or dimple, impact as well as sliding and tilting motion can occur. The more the rod's freedom of movement grows, the greater the 
variety of its possible movements and the more intense these movements become. The result is a greater susceptibility of the fuel rod to fretting, with the probability of fretting damage at a maximum.

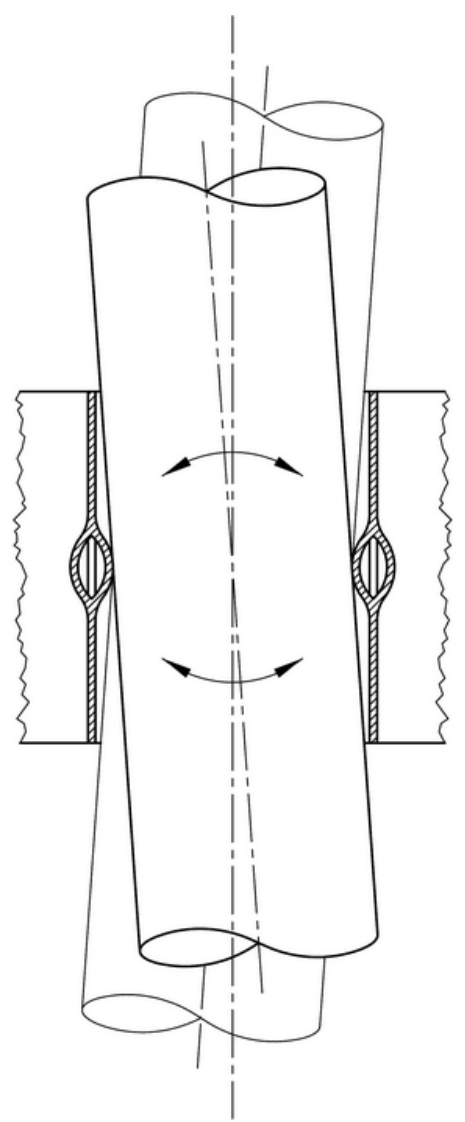

Fig. 1. Rod motion at BOL

Another factor of influence in connection with fretting is the geometry of the spacers [4,7]. The spacer grids consist of square cells with springs and dimples (dimples are much stiffer than springs) that position the fuel rods laterally. The number and shapes of these springs and dimples as well as their positions relative to each other affect the dynamic properties (e.g. resonant frequency and damping) of the vibrating rod/support system, and the intensity of fuel rod motion. These factors have a direct effect on the fretting susceptibility of the rod/support system. For example, the presence of edges at the point of contact, the geometries of these edges, and the stiffness of the two bodies in contact are of great significance.

AREVA NP has special fretting test stands (referred to below simply as "autoclaves") equipped with electromagnetic excitation systems for investigating the fretting behavior of rods. The primary task of these autoclaves is to deliberately induce fretting. The dynamic behavior of the vibrating system is recorded at the same time. The autoclaves are suitable for investigating all kinds of different fuel rod support configurations.

The aim of the work presented here was to investigate the susceptibility of the fuel rods to fretting under three different conditions of support ("Type A", "Type B" and "Type C"). Each of these support configurations had dimples and springs provid- ing eight points of contact with the test rod. The springs of the "Type A" and "Type B" supports have convex contours, while the "Type C" springs are concave. Each fretting test lasted for 1000 hours.

\section{Description of Tests}

Before the actual fretting tests were performed in the autoclave, measurements were taken under simulated flow conditions [8]. For this, the dynamic behavior of an entire fullscale fuel assembly was measured in a flow channel with water. This flow channel permits highly realistic simulation of the flow around a fuel assembly. The results obtained from the flow measurements (fuel rod vibration amplitude and frequency) served as a basis for the excitation signal used in the autoclave tests. The excitation signal with the same spectrum and with the same intensity was used for all test rods to ensure comparability of the results. Only mock-ups were used for the supports in the fretting tests described here.

Unlike the flow measurements, the autoclave tests were performed on a single shortened-length test rod. A wide range of supports and fits can be configured in the autoclave to study the fretting susceptibility of various fuel rod support configurations. The fretting tests in the autoclave can be interrupted at any time for inspections. The aim of these is to establish the current condition of support (determine the so-called "clear" inside diameter of the support cell) and characterize the fretting marks produced on the test rod.

\subsection{Autoclave}

Fig. 2] shows the configuration of the autoclave with its main components. The test rod in the autoclave can be excited by means of electromagnet in the lateral direction by the EMAC (Electromagnetic Actuation Control) system located outside of the Plexiglas box. The EMAC system can induce vibration of the test rod such as that which occurs in a real reactor. The current intensity of the EMAC system's electromagnetic coils is proportional to the excitation force acting on the rod. The rod's lateral oscillations can be measured using the displacement sensors mounted in the wall of the Plexiglas box. The azimuthal stabilization system prevents uncontrolled rotation of the rod about its longitudinal axis. The test rod is suspended from a thin wire and supported at three elevations along its axis. The fretting tests can be performed in air or in water.

\subsection{Test Specimens}

Zirconium-alloy cladding tubes manufactured for actual fuel assemblies were used for the tests. Instead of uranium pellets, the cladding tubes were filled with molybdenum cylinders. To ensure reliability and reproducibility of the tests, the specifications regarding straightness of the test rod and fit were strictly checked and exactly adhered to.

Two of the investigated support configurations ("Type A" and "Type B") are of similar design, with four convex spring ele- 


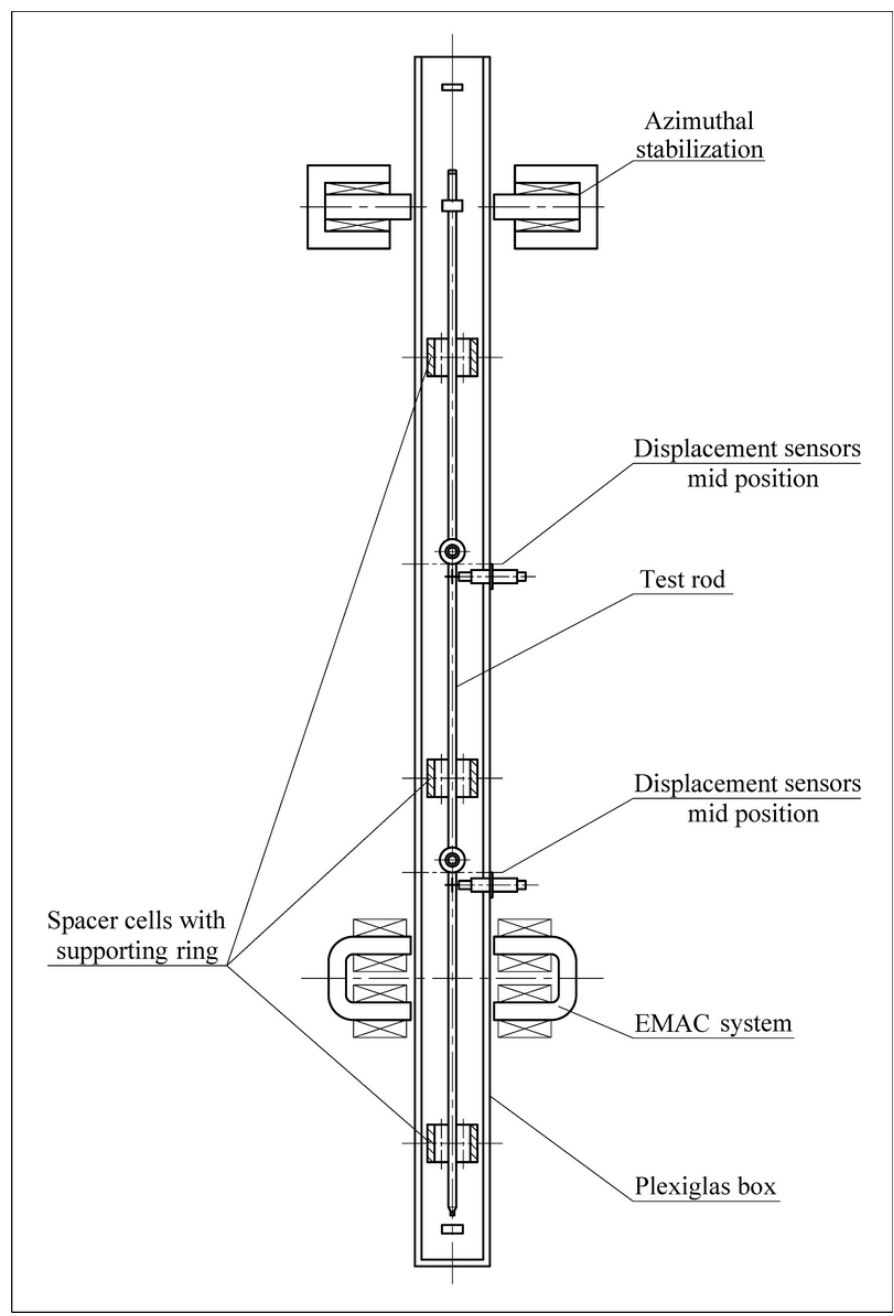

Fig. 2. Configuration of autoclave

ments. The surface area of the spring in contact with the fuel rod is larger in "Type A" than in "Type B". The edges of "Type A" and "Type B" differ in terms of their shapes and locations. The heavily rounded edges of "Type A" have no contact with the rod surface. In contrast to the "Type A" design, edge contact with the rod can potentially occur in the "Type B" support, especially when the springs have suffered considerable wear. The "Type C" configuration also investigated here differs from the other two by having concave contours. Unfortunately, figures of the support types cannot be included due to copyright reasons.

One test was performed for each of the three support types using the same initial grid-to-rod gap $0.10 \mathrm{~mm}$ at all three axial support elevations. These tests were supplemented by an additional test carried out on the "Type A" support using a different gap: unlike in the other tests, a gap of $0.05 \mathrm{~mm}$ (instead of $0.10 \mathrm{~mm}$ ) was provided at the bottom support in this test. The gap conditions at the three support elevations are $0.10 / 0.10 / 0.10 \mathrm{~mm}$ and $0.05 / 0.10 / 0.10 \mathrm{~mm}$ signed from the bottom to the top elevations, respectively.

\subsection{Test Procedure}

All of the fretting tests were performed in water at room temperature for 1000 hours. The tests were interrupted at certain intervals so that the fretting marks could be measured. During each inspection, the number and depth of the fretting marks of the impressions were measured using an interferometer. The interferometer scanned the entire rod surface and generates a three-dimensional image of the worn surface. A computer program developed for this purpose determined an ideal cylindrical surface based on the undamaged measured surface. In the second step the program evaluated the volume (which is the volume of the measured mark) between the ideal cylindrical surface and the real measured fretted surface of the test rod.

Rod vibration was recorded during the fretting tests so that the change in vibration behavior over time could be traced more easily. To study the dynamic behavior of the rod/support system (resonant frequency and vibration amplitude), displacement sensors were used to measure lateral movements of the test rod in and perpendicular to the direction of excitation. These vibration measurements were taken before and after each fretting test as well as at each interruption for inspecting the fretting marks. The spectrum of the excitation signal applied to the rod in both the fretting tests and the vibration measurements had a frequency range of $5 \mathrm{~Hz}$ to $80 \mathrm{~Hz}$.

\section{Results}

\subsection{Fretting Volume}

In terms of energy, vibration of the test rod was induced by means of electromagnet during the tests by the excitation system. The input energy of the rod was dissipated by damping mechanisms of the water/rod/support system (liquid damping, material damping, friction and impact) and by the particle detachment during the fretting process. The aggregate fretting volume (sum of the entire volume of fretting marks on the test rod and the rod supports) is a parameter that can be used to characterize the energy dissipated by the process of fretting. Fig. 3 shows the aggregate fretting volume as a function of test time.

The aggregate fretting volume in the "Type A" support configuration is much smaller than in "Type B" and "Type C". Unlike "Type A", the "Type B" and "Type C" supports have edges at the point of rod-to-support contact and are in contact with the test rod over a smaller surface area. Although these edges are rounded, high stress concentrations arise in the material at the point of contact between edge and rod, resulting in greater wear of the rod surface.

The curves of the aggregate volumes in the various support types differ significantly from one another. In both tests conducted with the "Type A" configuration, the aggregate volume increased linearly as a function of test time. In contrast to "Type A", the "Type B" configuration initially exhibits a slightly degressive and later a slightly progressive behavior. The "Type C" support exhibits degressive behavior. When the two "Type A" tests are compared, it can be seen that although the behavior of the volume curves is the same, differences can be found in the fretting rate and the aggregate volume. The additional test with the smaller gap $(0.05 / 0.10 / 0.10 \mathrm{~mm})$ exhibits a 


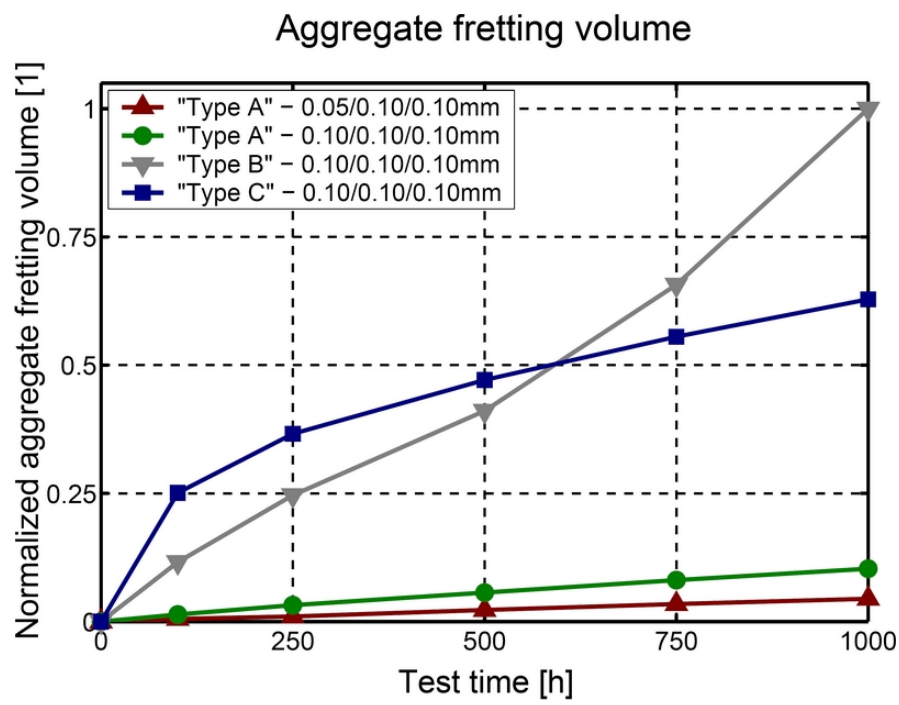

Fig. 3. Aggregate fretting volume

lower fretting rate as well as a smaller aggregate volume.

In contrast to "Type B", the "Type C" configuration has springs with concave contours, and edges that do not run parallel to the rod axis. These differences also explain the different fretting curves of the two types of support.

\subsection{Contact Geometry}

To describe the spring contact area the following parameters should be given consideration with regard to rod fretting: contact area of individual springs $\left(A_{i}\right)$, and length $\left(l_{i}\right)$ of spring edges in contact. For further analysis, a design factor is defined here which describes the ratio of edge length to contact area:

$$
D_{i}=\frac{l_{i}}{A_{i}}
$$

As the ratio of spring edge length to contact area varies significantly depending on the type of spring, the design factor alone cannot be used to characterize spring types. In addition to the design factor, the edge geometry and edge orientation relative to the rod axis must also be taken into account. As fretting progresses, not only does the geometry (shape) of the fretting mark change, but also that of the spring contact area. This means that the design factor is not a constant parameter but changes in the course of the fretting process. The design factor represents one means for assessing the effect of the spring edges contributing to fretting.

The size of the contact area as well as the way it increases differ from support type to support type. In "Type A", the contact quickly develops from initially line-type contact to area contact. Because the contact is between two convex bodies (spring and rod), the rate of growth decreases over the course of fretting. In contrast to "Type B" and "Type C", the "Type A" configuration does not have any spring edge at the point of contact, with the result that the value of the design factor remains zero during the entire fretting process.

In the case of "Type B" there is also initially line contact which quickly develops, however, into area contact due to the edges with their heavy wearing effect. In contrast to "Type A", the springs in "Type B" are much narrower, with the result that the springs already touch the rod widthwise at the start of the fretting process but not lengthwise. As fretting progresses, the length of the edges in contact increases in a similar way to the fretting area. For this reason the design factor for "Type B" only changes within a small range.

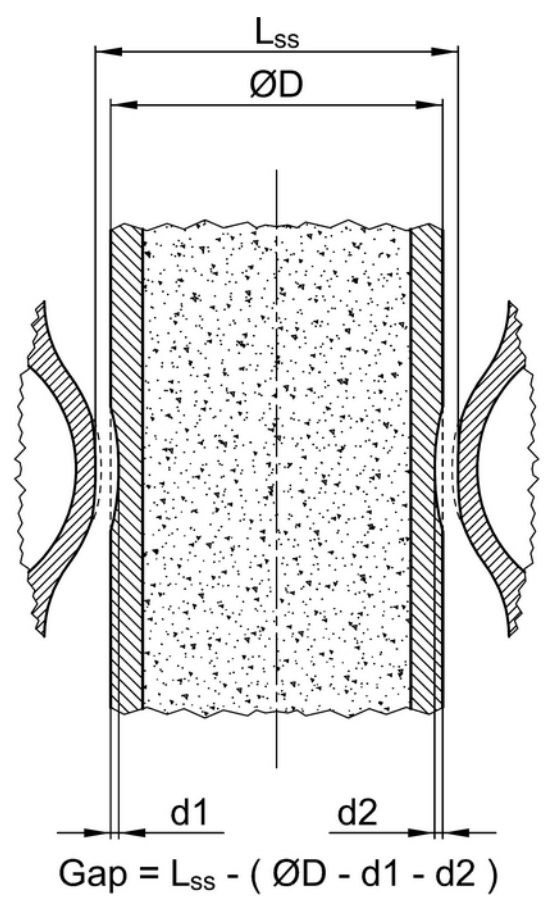

Fig. 4. Determination of support-to-rod gap

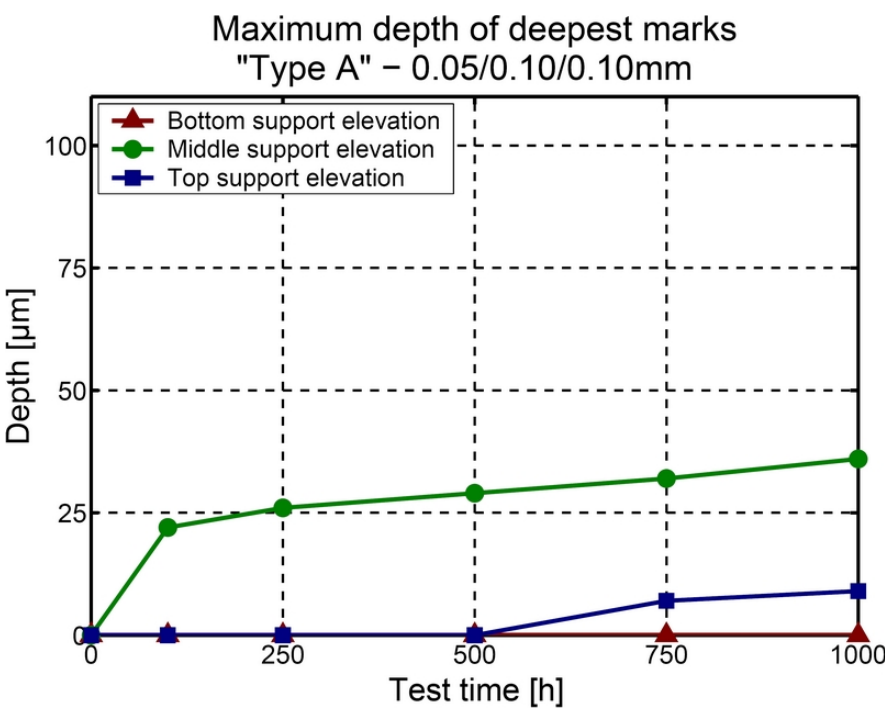

Fig. 5. Maximum depth of deepest marks "Type A"- $-0.05 / 0.10 / 0.10 \mathrm{~mm}$

As the spring edge of "Type C" (with concave contours) is not parallel to the rod axis, only a small part of it is in contact with the surface of the rod. At the start of the test there is line contact, just as in "Type A" and "Type B". The contact area and the length of the edge in contact increase sharply over the course of the test due to the convex/concave contact. Due to the geometry of the spring, the contact area and the length of the spring edge that is in contact increase at the same rate. During the fret- 


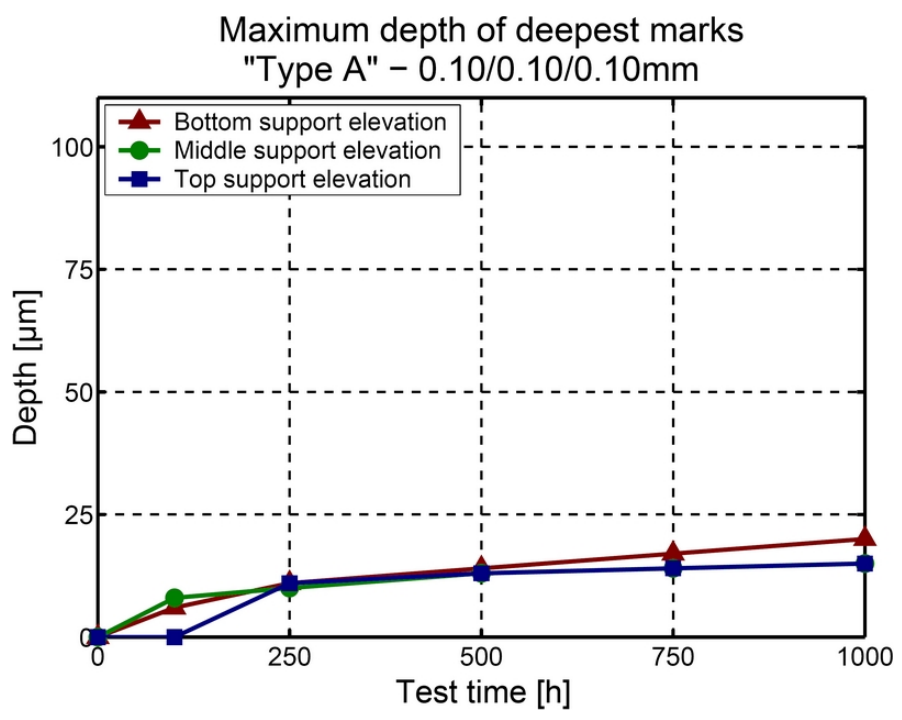

Fig. 6. Maximum depth of deepest marks "Type A" - 0.10/0.10/0.10 mm

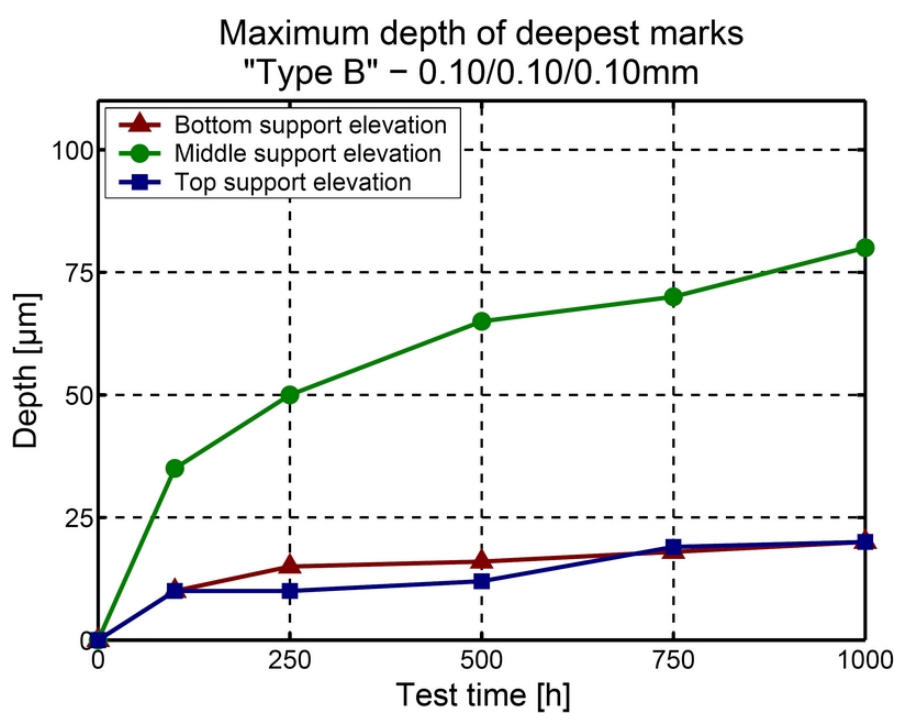

Fig. 7. Maximum depth of deepest marks "Type B" $-0.10 / 0.10 / 0.10 \mathrm{~m}$

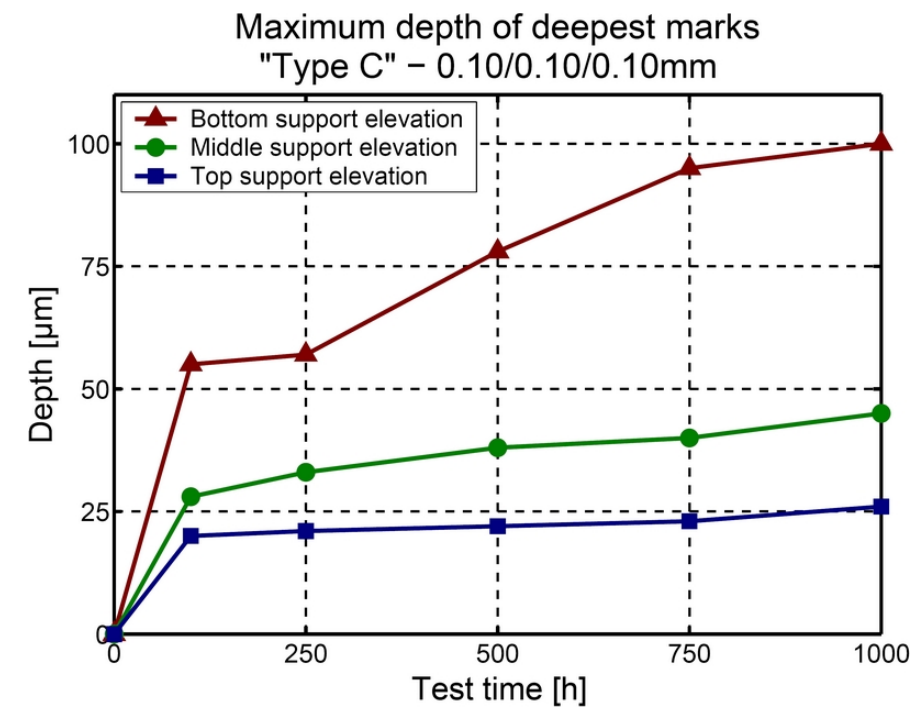

Fig. 8. Maximum depth of deepest marks "Type C" - 0.10/0.10/0.10 mm

ting process, the design factor for "Type C" remains constant, as in the case of "Type B", but is smaller. The growth rate of the contact area is higher in the case of convex/concave contact than with convex/convex contact. The length of the spring edges in contact in "Type C" is much shorter than in "Type B". The degressive volume curve of "Type $\mathrm{C}$ " can be explained by the contact pressure, which is initially high and decreases over the course of the test, and by the convex/concave contact geometry.

In all support configurations, the contact pressure decreases as the contact area grows, assuming that the applied load remains the same. Decreasing growth of the contact area reduces the rate at which the contact pressure decreases.

\subsection{Fretting Depth and Grid-to-Rod Fit}

The most important parameter in connection with fretting as far as the operating reliability of the fuel assembly is concerned is the depth of the fretting marks. One of the factors that has the greatest influence on the development of fretting is the fit between the support elements in the spacer grid and the fuel rod.

The gap at this location, which is directly correlated to the fretting mark depth, increased during the fretting tests. Support element wear also has an influence on this gap, although to a considerably lesser degree, something which depends on the support type.

To determine the support-to-rod gap during the fretting tests, measurements were taken of the depth of the fretting marks on the rod and of the distances between the opposing springs (Fig.47). As there are two pairs of opposing springs at each axial support elevation, two gap dimensions result on account of the associated rod fretting marks.

Fig. 5 to 8 show the change in the depth of the fretting marks over time for all three types of support. Each diagram shows the depth curves for the individual support elevations based on measurement of the deepest marks. As the figures show, "Type C" exhibits the deepest fretting marks and "Type A" the shallowest. The figure for the "Type A" configuration, in which the initial gap was the same at all elevations $(0.10 / 0.10 / 0.10 \mathrm{~mm})$, shows that the marks develop to a similar extent at all three elevations (Fig. 6). In the tests with the other types of support the fretting marks developed at the three elevations different.

Fig. 9 to Fig. 12 show the development of the support-to-rod gap over time for all three support types. Each diagram shows the time histories of the gap for the individual support elevation based on the maximum gap measured.

The greatest increase in clearance was found in "Type C". The gap in "Type A" changed the least. The gap in the fretting test conducted with the "Type C" configuration increased sharply during the first $100 \mathrm{~h}$. After $100 \mathrm{~h}$, it continued to grow but to a much lesser extent. The curve is approximately linear in this section. As in the "Type C" configuration, the gap in "Type B" also exhibits the highest growth rate during the first $100 \mathrm{~h}$. After $100 \mathrm{~h}$, it grows less quickly but follows an approximately linear curve. As the curves show, growth rate is higher in the "Type C" configuration during the first $100 \mathrm{~h}$ than in "Type B". After $100 \mathrm{~h}$, this tendency changes. 
Maximum gap

"Type A" - 0.05/0.10/0.10mm

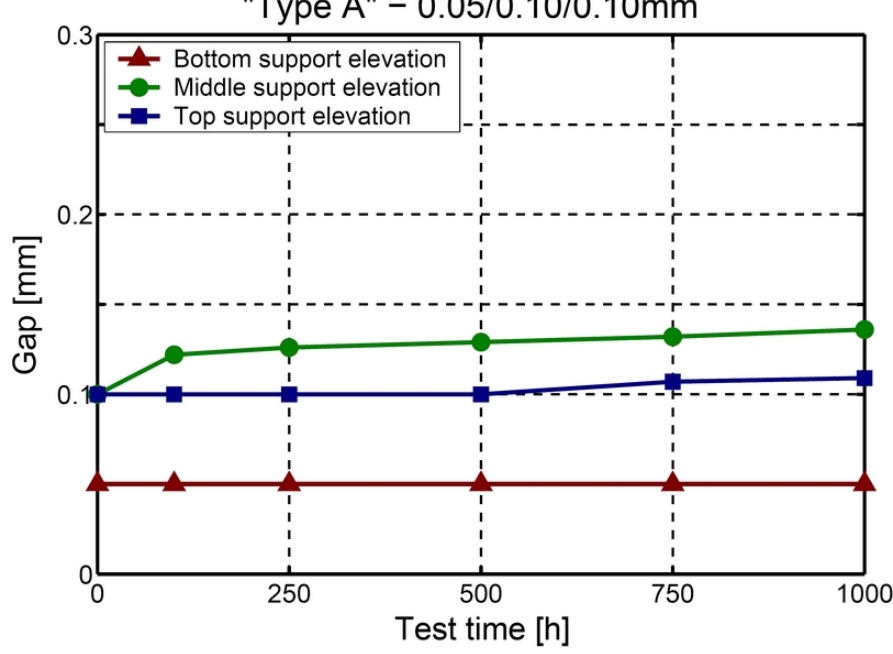

Fig. 9. Maximum gap, "Type A" $-0.05 / 0.10 / 0.10 \mathrm{~mm}$

Maximum gap

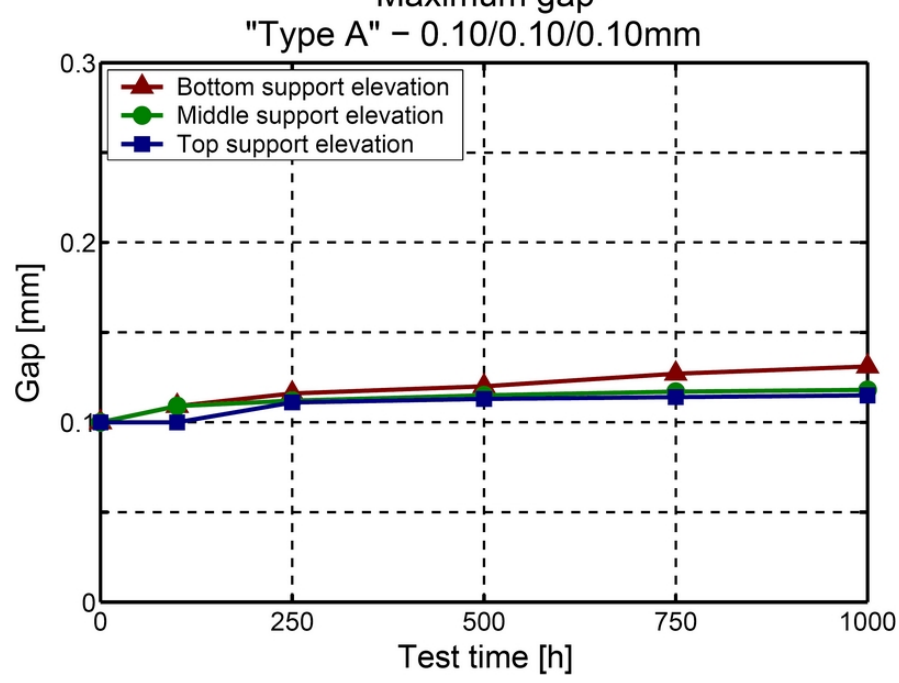

Fig. 10. Maximum gap, "Type A" $-0.10 / 0.10 / 0.10$ mm

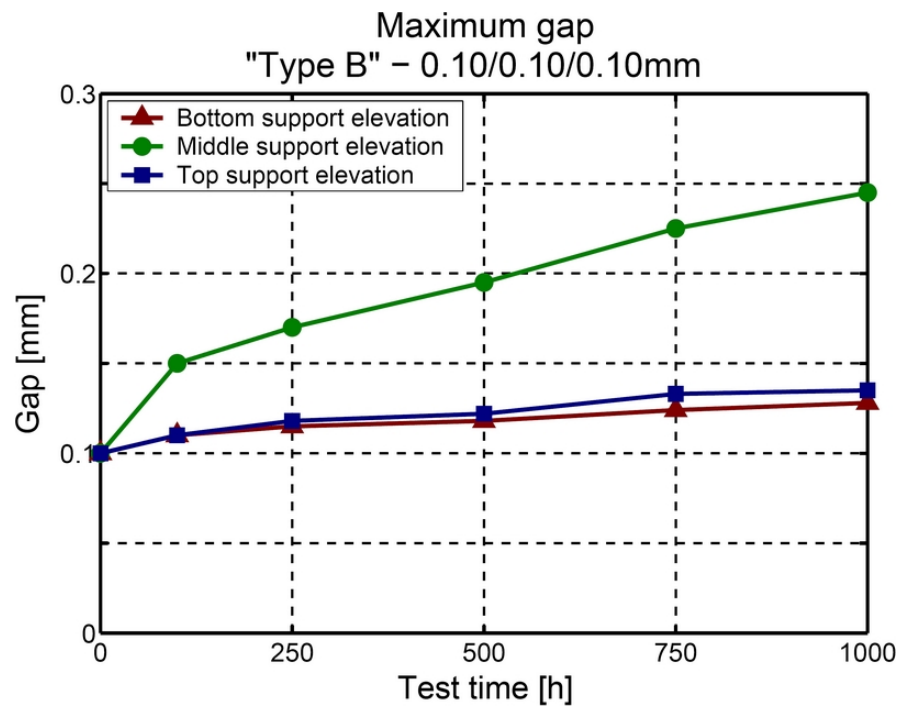

Fig. 11. Maximum gap, “Type B" $-0.10 / 0.10 / 0.10$ mm

Both gaps increase approximately linearly, but the growth rate in "Type B" is higher than in "Type C". The curve of the "Type A" gap is similar to that of "Type B" and "Type C". The

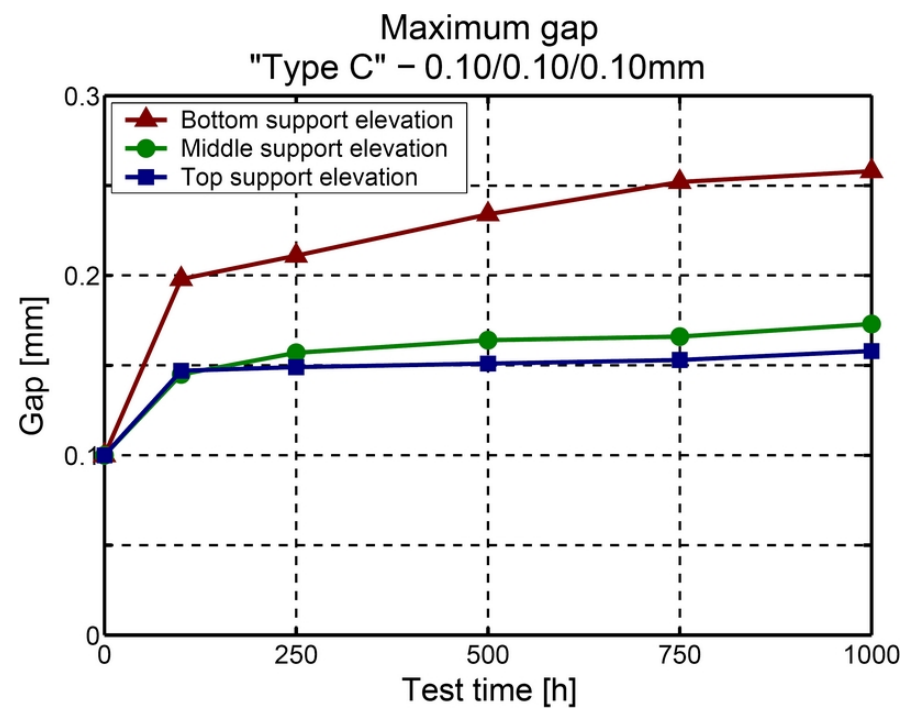

Fig. 12. Maximum gap, "Type C" $-0.10 / 0.10 / 0.10$ mm

Transfer functions "Type A" - 0.05/0.10/0.10mm

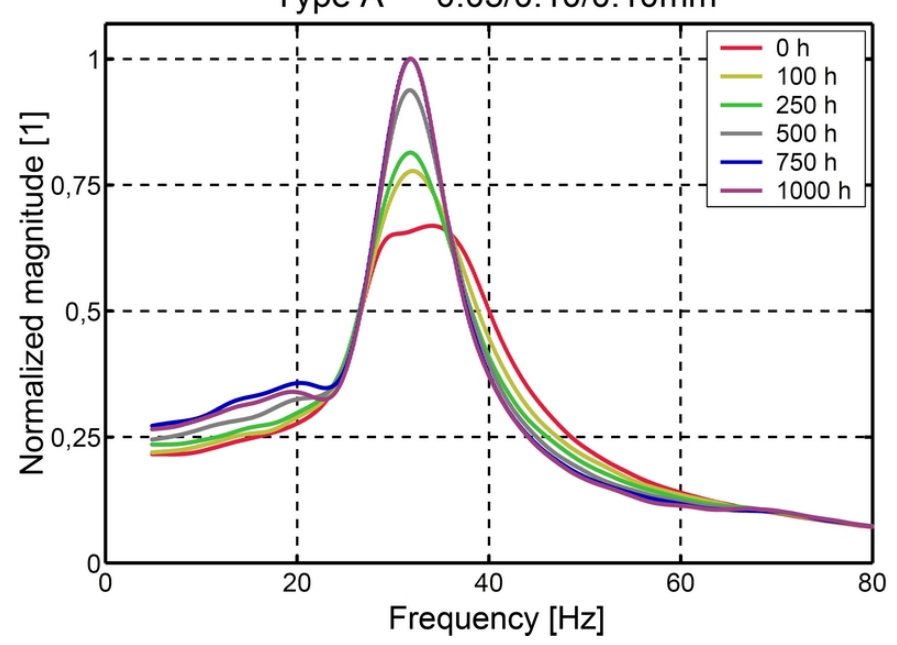

Fig. 13. Transfer functions, “Type $A$ " $-0.05 / 0.10 / 0.10 \mathrm{~mm}$

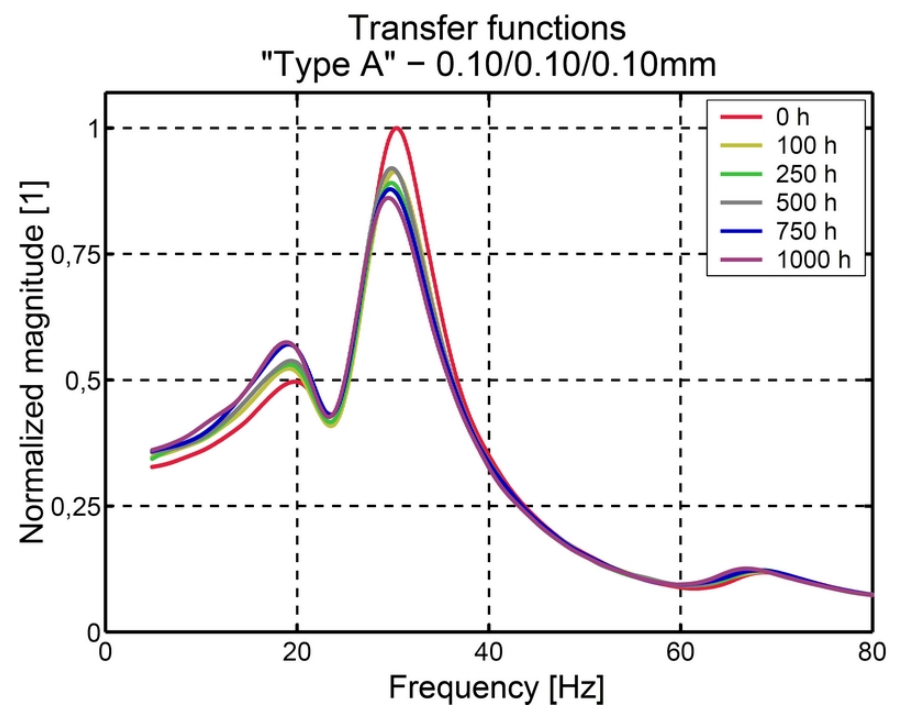

Fig. 14. Transfer functions, "Type $A "-0.10 / 0.10 / 0.10 \mathrm{~mm}$

gap increases much more quickly during the first $100 \mathrm{~h}$ than at later times. However, the gaps and the growth rates are much smaller than in the other support configurations. 


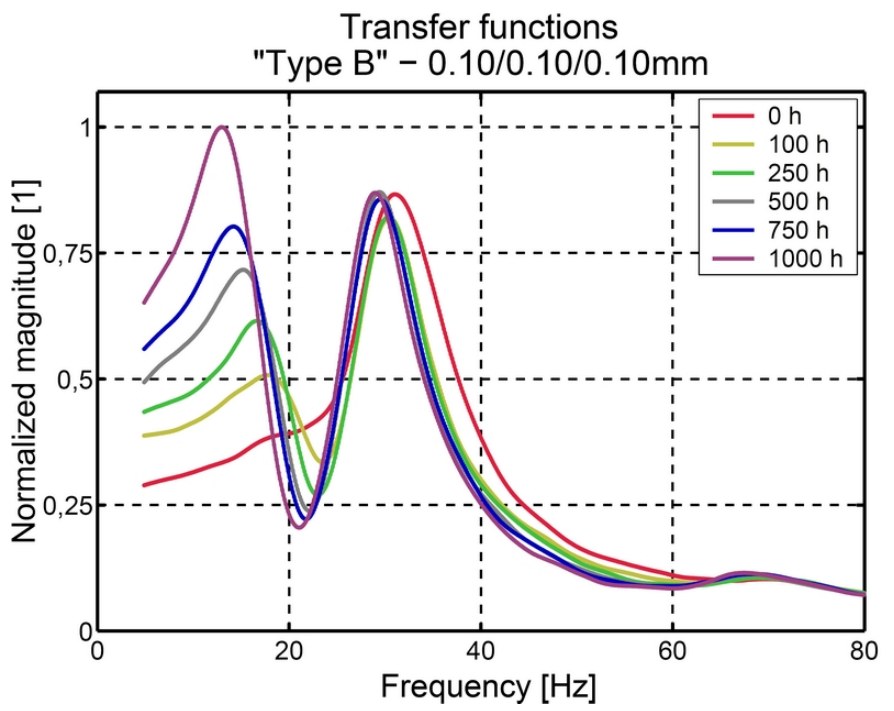

Fig. 15. Transfer functions, "Type B" $-0.10 / 0.10 / 0.10 \mathrm{~mm}$

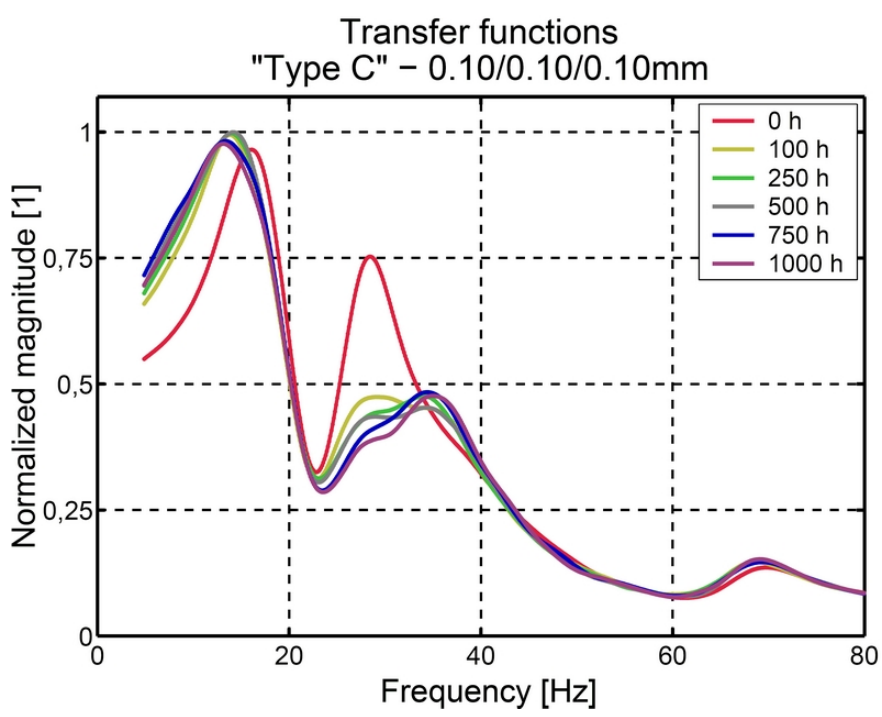

Fig. 16. Transfer functions, "Type C" $-0.10 / 0.10 / 0.10 \mathrm{~mm}$

The change in fretting mark depth as well as in the supportto-rod gap is governed by the size of the contact area. The line contact existing between the rod and the support spring at the start of the test induces a high stress concentration in the material, which initially results in the fretting marks quickly becoming deeper. The fast growth in contact area, however, slows down the rate of growth in fretting mark depth and support-torod gap.

As the gap becomes larger, the rod moves more freely and more extensively at the same level of excitation. This can result in an increase in fretting rate. For example, in Fig. 3 it can be seen that the growth rate of the aggregate fretting volume increases after $500 \mathrm{~h}$ in the "Type B" configuration. In summary, it can be said that knowledge of how the grid-to-rod gap behaves is indispensable for understanding the fretting process.

\subsection{Vibration Analysis}

Fuel rod motion, which is governed by the input excitation and by the dynamic properties (resonant frequency and damping) of the vibrating rod/support system, is the cause of fretting.
The dynamic properties of the vibrating system depend on the support and the fit. Vibration measurements were taken to study the system's dynamic properties.

Lateral movements of the test rod were measured in and perpendicular to the direction of excitation between the top and middle supports (top span) and between the middle and bottom supports (bottom span).

Investigation of dynamic properties is based on the transfer function of the vibrating system. The transfer function was calculated using the excitation current as the system input and rod movement in the bottom span in the direction of excitation as the system output. Knowledge of rod motion close to the excitation system is necessary in order to be able to monitor how the energy that is input into the vibrating system changes over time. For this reason, only rod motions in the bottom span in the direction of excitation were considered for the transfer function.

Fig. 13 to 16 show the transfer functions at different points in times during each of the four fretting tests. The curves of the transfer function for the additional test ("Type A" support, initial gap: $0.05 / 0.10 / 0.10 \mathrm{~mm}$ ) change with time. The growth of the resonance peak at $32 \mathrm{~Hz}$ reaches a maximum during the first $100 \mathrm{~h}$. After $100 \mathrm{~h}$, this growth diminishes up to a test time of $750 \mathrm{~h}$. Between $750 \mathrm{~h}$ and $1000 \mathrm{~h}$, growth of the resonance peak is nearly zero. Growth of the resonance peak is related to the reduction in damping.

The behavior in the fretting test conducted with the "Type A" support (initial gap: $0.10 / 0.10 / 0.10 \mathrm{~mm}$ ) is similar to that in the additional test ("Type A" support, initial gap: $0.05 / 0.10 / 0.10 \mathrm{~mm})$. The change in the transfer function with time is largest during the first $100 \mathrm{~h}$. The changes are smaller, however, than those of the additional test conducted with the “Type A" support (initial gap: 0.05/0.10/0.10 mm).

In contrast to the tests with the "Type A" configuration, the "Type B" and the "Type C" supports exhibit a pronounced change in the transfer function with increasing test time. The reason for this is the growth of the support-to-rod gap, which is considerably larger than in "Type A".

For all three support configurations it can be said that the change in the transfer function over time is similar to that of the rod-to-support gap. This correlation is most clear to see during the first $100 \mathrm{~h}$.

In the first $100 \mathrm{~h}$, not only does the gap increase much more than at other times, but the transfer function also changes significantly. The following now addresses the behavior of the transfer function and of the rod-to-support gap in the time between $100 \mathrm{~h}$ and $1000 \mathrm{~h}$. The change in the transfer function and in the gap during this period of time is smaller than during the time from $0 \mathrm{~h}$ to $100 \mathrm{~h}$. Compared to the "Type A" and "Type C" configurations, the time history of the "Type B" transfer function and gap exhibits the greatest change between $100 \mathrm{~h}$ and $1000 \mathrm{~h}$.

The growth of the gap also causes a reduction in the stiffness of the vibrating system. In summary it can be said that, in the case of the supports with an initial gap of $0.10 / 0.10 / 0.10 \mathrm{~mm}$, 
the first resonant frequency decreases as test time progresses, with the magnitude increasing at the same time. In contrast, the additional test with an initial gap of 0.05/0.10/0.10 mm exhibits a different behavior with regard to the first resonant frequency. In the course of the additional test, the first resonant frequency develops within the range of 15 to $20 \mathrm{~Hz}$. This resonance curve describes the rigid-body motion of the rod.

Following this qualitative description of rod motion using the transfer function, a quantitative description is now given based on the effective vibration amplitudes, commonly referred to as the root-mean-square (RMS) vibration amplitudes. Figures 17 to 20 show the RMS vibration amplitudes for the various types of support. Each diagram contains the vibration amplitude curves of the four rod motion signals measured for each type of support.

During the additional test ("Type A", initial gap: $0.05 / 0.10 / 0.10 \mathrm{~mm}$ ) the intensity of rod motion increased slightly over the course of the test time. This applies to all four measurement signals. The diagram for "Type A" with an initial gap of $0.10 / 0.10 / 0.10 \mathrm{~mm}$ shows that the intensity of motion is approximately constant in the case of all four measured signals. The vibration amplitude curves for "Type B" can be divided into two groups as regards their behavior. Major differences are evident between the intensity curves for motion in and perpendicular to the direction of excitation. While the intensity in the direction of excitation increases continuously and significantly over time, the intensity of motion perpendicular to the direction of excitation exhibits a considerably smaller but steady growth. The "Type C" support exhibits a pronounced change in the intensity of rod motion during the first $100 \mathrm{~h}$. Only a slight increase in the intensity of rod motion can be seen to occur in the time from $100 \mathrm{~h}$ to $1000 \mathrm{~h}$.

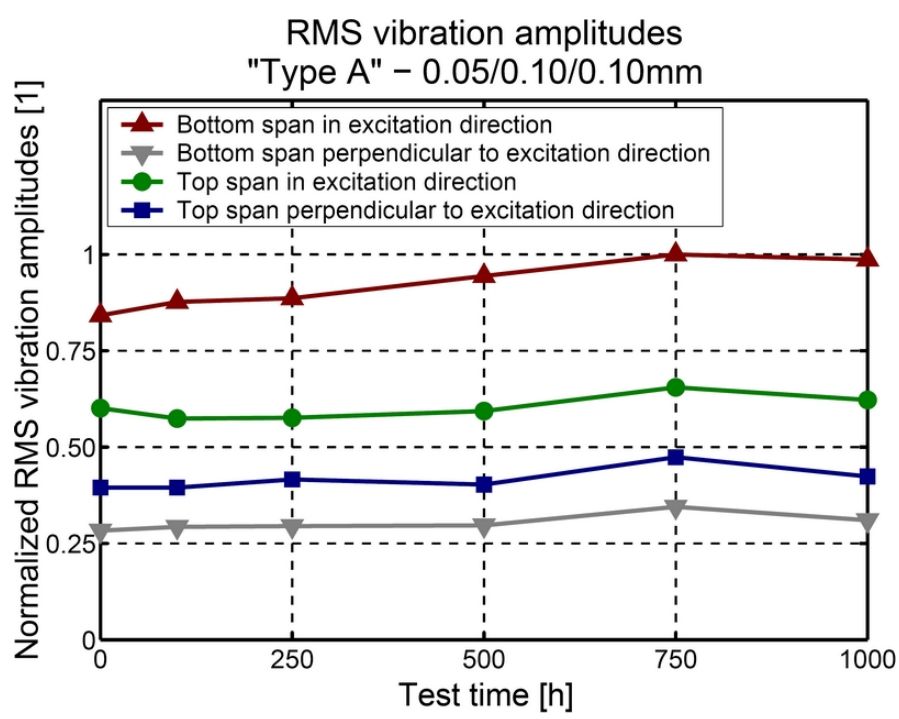

Fig. 17. RMS vibration amplitudes, "Type $A "-0.05 / 0.10 / 0.10 \mathrm{~mm}$

The increase in motion intensity can be attributed to growth of the rod-to-support gap. At the same level of excitation, the intensity of motion increased when the gap increased. The largest increase in RMS vibration amplitude occurred in the test con-
RMS vibration amplitudes

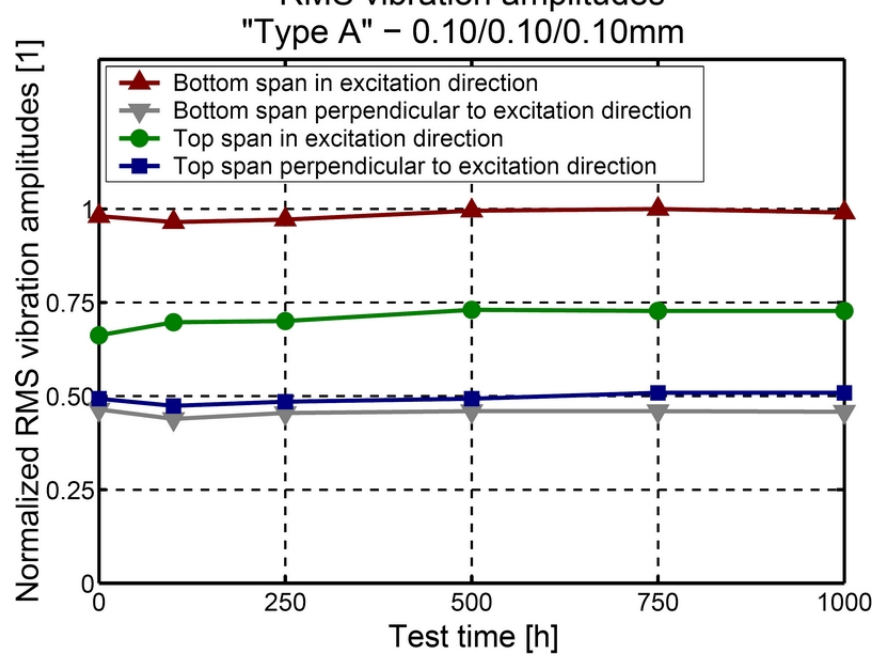

Fig. 18. RMS vibration amplitudes, "Type A" - 0.10/0.10/0.10 mm

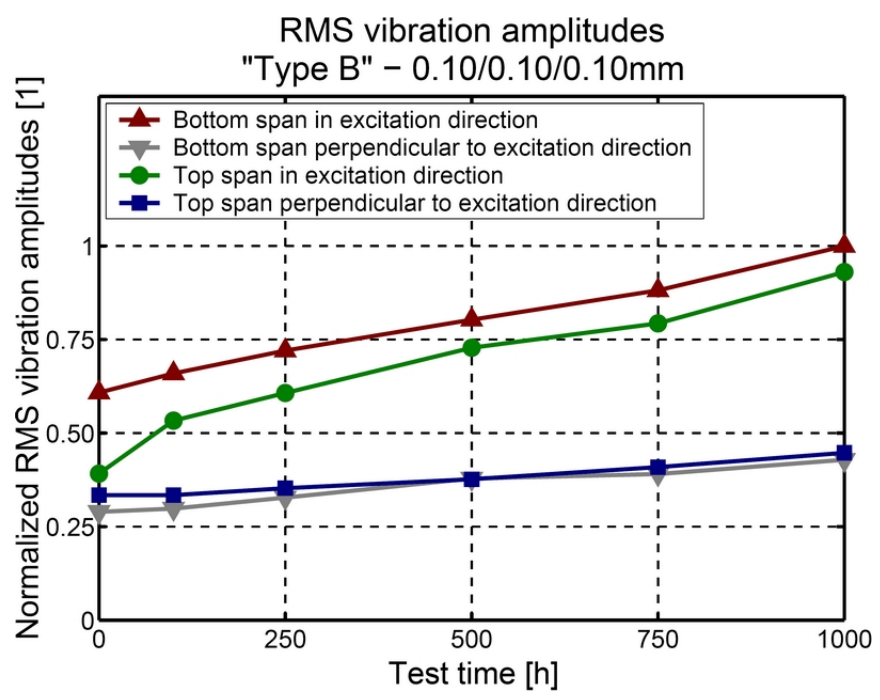

Fig. 19. RMS vibration amplitudes, “Type B" - 0.10/0.10/0.10 mm

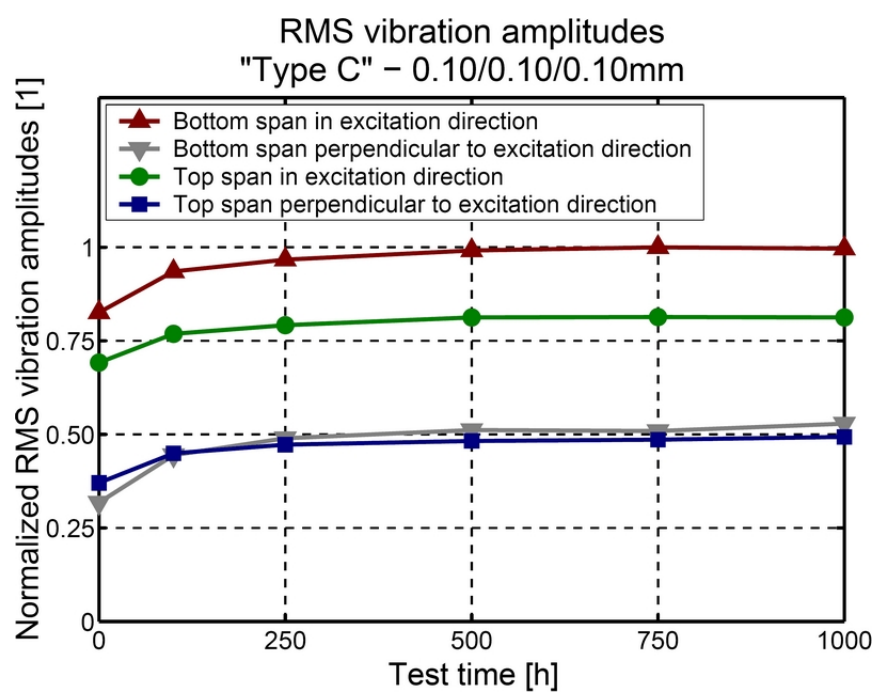

Fig. 20. RMS vibration amplitudes, "Type C" $-0.10 / 0.10 / 0.10 \mathrm{~mm}$

ducted with the "Type B" support. Fig. 11 shows that the growth rate of the largest gap was high during the entire test.

As can be seen from the figures, the large changes in the rod motion intensity correspond to the large changes in the transfer function. This relationship is most clearly evident in the 
test with the "Type B" support. Another example of the parallel changes occurring in motion intensity and transfer function can be seen in all of the tests during the first $100 \mathrm{~h}$, during which time - as was already described - the most pronounced changes take place.

\section{Model}

The preceding sections showed that the fretting process is complex and that the aggregate fretting volume, the fretting mark depth and the rod-to-support gap, as well as the dynamic behavior and intensity of rod motion, all interact with each other. The prime cause of fretting is rod movement, this being dependent on the type of support as well as on the rod-to-support gap. In turn, the fretting or wear occurring on the rod and on the support have a direct effect on the rod-to-support gap.

Because of the significant differences in the fretting process that are shown to exist up to a test time of $100 \mathrm{~h}$ and after 100 $h$ by the transfer functions, the rod-to-support gap and the intensity of rod motion, the fretting process is divided into two phases. The phase leading up to a test time of $100 \mathrm{~h}$ that experiences the largest changes is designated the starting phase. The differences between the individual support configurations can be seen most clearly in the second phase. Aggregate fretting volume increases linearly in the case of "Type A" and degressively in the case of "Type C". The "Type B" configuration first exhibits a degressive increase in fretting and later a progressive increase.

The aggregate fretting volume represents the energy dissipated by the fretting process. The fretting volume $d V(t)$ arising during a small time interval $d t$ can be illustrated as a function of the energy $d W(t)$ dissipated during the fretting process.

$$
\frac{d V(t)}{d t}=k_{\text {wear }}(t) \cdot \frac{d W(t)}{d t}
$$

The overall wear coefficient $k_{\text {wear }}(t)$ accounts for all wear mechanisms that contribute to fretting (such as adhesion, abrasion, corrosion etc.). The aggregate fretting volume is determined by integrating equation (Eq. 2). The aggregate fretting volume increases linearly with increasing test time if the following conditions are fulfilled: the relative contributions made by the individual wear mechanisms must not change during the test, and the fraction of the rod's kinetic energy that goes into fretting must remain constant.

If the design factor (Sec. 3.2) and the rod-to-support gap (Sec. 3.3) remain constant, then neither will the ratios of the wear mechanisms to each other or the overall wear coefficient $k_{\text {wear }}$ change. The design factor describes the relationship between line-type contact and area contact. The change in the gap is an indication of the change in the impact energy in the case of impact wear. If the design factor and the gap do not change significantly during the fretting test, then the qualitative condition for steady-state wear is met: namely, the overall wear coefficient $k_{\text {wear }}$ is non-time-dependent.
The quantitative condition for steady-state wear - namely, that the fraction of the rod kinetic energy that is dissipated by fretting remain constant during the test - is fulfilled if the damping of the vibrating rod/support system and the energy input by the excitation system into the vibrating system remain constant. This can be determined by analyzing rod motion. If the transfer function describing the dynamic behavior of the vibrating rod/support system and the intensity of rod motion do not change significantly during the fretting process, this is an indication that the energy input by the excitation system into the vibrating system and the damping of the vibrating system are remaining constant. When all of the aforementioned conditions are met, not only does the energy input into the system remain constant during the test but also the energy dissipated by the fretting process.

The outcome of these considerations is that fretting is steadystate. The fretting rate $(\dot{V})$ can therefore be calculated as follows:

$$
\dot{V}=k_{\text {wear }} \cdot \dot{W},
$$

where $\dot{W}$ denotes the energy dissipated per unit time.

In this study, the fretting test of the "Type A" support (initial gap: $0.10 / 0.10 / 0.10 \mathrm{~mm}$ ) fulfills all conditions for steady-state fretting. The curve of the aggregate fretting volume confirms that fretting increases linearly with time.

\section{Conclusions}

The effect of test time on rod fretting behavior was investigated for three different rod support mock-ups. The fretting tests were carried out in an autoclave with electromagnetic excitation in water at room temperature. The depth of the fretting marks and their volume increased as testing progressed.

It was found that the fretting process has a starting phase that ended after a test time of 100 hours in the tests described here. After this starting phase, the fretting process was dependent on the type of support configuration.

The resulting increase in the grid-to-rod gap caused changes in rod dynamic behavior and in the intensity of rod motion. Fretting rate is affected by rod motion, and the presence of edges at the point of contact between rod and support accelerates fretting wear. Spring design affects not only the degree of fretting but also the time history of the fretting process.

Tests (both convex and concave support contours) conducted with edges at the point of contact between rod and support exhibit heavy fretting. Because of the beneficial convex/concave contact, aggregate fretting volume increases degressively in the test with concave support contours.

The requisite conditions for steady-state fretting were identified:

1 The proportional contributions of the wear mechanisms taking part in the fretting process must remain constant during the test.

2 The fraction of the rod's kinetic energy that is dissipated by the fretting process must remain constant during the test. 
Steady-state fretting was identified in the case of rods supported by convex springs without edges at the point of rod-to-support contact.

However, it must not be forgotten that the results presented here are based on model tests. These results can serve as a basis for the future development of spacer grids with an even higher degree of fretting resistance. The process of fretting taking place in a real reactor is, however, far more complex than the behavior that can be simulated in the autoclave. But, although the autoclave tests are not adequate for faithfully modeling the real processes, they do contribute towards a better understanding of the fretting process.

\section{References}

1 Csom Gy, Atomerómúvek üzemtana, II. kötet Az energetikai atomreaktorok üzemtana, Múegyetemi Kiadó, 2005.

2 Pettigrew M.J, Taylor C.E, Fisher N.J, Yetisir M, Smith B.A.W, Flowinduced vibration: recent findings and open questions, Nuclear Engineering and Design, 185, (1998), 249-276, DOI 10.1016/S0029-5493(98)00238-6

3 OECD: Glossary of Terms and Definition in the Field of Friction, Wear and Lubricant, OECD Publications; Paris, 1969.

4 Kim H.K, Lee Y.H, Influence of contact shape and supporting condition on tube fretting wear, Wear, 255, (2003), 1183-1197, DOI 10.1016/S00431648(03)00068-1

5 Kovács Sz, Stabel J, Ren M, Ladouceur B, Influence of Grid-to-Rod Fit on Fuel Rod Fretting, Periodica Polytechnica. Article in press.

6 Billerey A, Evolution of fuel rod support under irradiation - Impact on the mechanical behaviour of fuel assemblies, Structural behaviour of fuel assemblies for water cooled reactors, (2005), 101-111. in Cadarache, France, 22-26 November 2004,IAEA-TECDOC-1454 Vienna.

7 Kovács Sz, Stabel J, Ren M, Ladouceur B, Comparative study on rod fretting behavior of different spacer spring geometries, Wear, 266(1-2), (2009), 194-199, DOI 10.1016/j.wear.2008.06.010

8 Stabel J, Koebke K, Stephens LG, Experimental and Analytical Procedures for Approving Fretting-Free Fuel Rod Support, (1999). LWR Conference Utah. 